\title{
A FLAT HIGH-FREQUENCY SCANNING MICROMIRROR
}

\author{
Robert A. Conant, Jocelyn T. Nee, Kam Y. Lau, and Richard S. Muller \\ Berkeley Sensor \& Actuator Center, University of California, Berkeley \\ Berkeley, CA 94720-1774
}

\begin{abstract}
We present the Staggered Torsional Electrostatic Combdrive (STEC) fabrication process that creates thick mirrors with hightorque actuators, thereby allowing high-speed optical scanning with large angular deflections. We have demonstrated a $550 \mu \mathrm{m}$ diameter mirror capable of scanning an optical angle of $24.9^{\circ}$ at its $34 \mathrm{kHz}$ resonant frequency with less than $30 \mathrm{~nm}$ static and dynamic deformation. The optical resolution is 350 pixels - very near the diffraction-limited resolution of 355 pixels with $655 \mathrm{~nm}$ wavelength light.
\end{abstract}

\section{INTRODUCTION}

Scanning mirrors are used in many applications: barcode readcrs, laser printers, confocal microscopes, and fibcr-optic network components. There are significant limitations to the performance of macro-scale scanners; in particular, their scanning speed, power consumption, cost, and size often preclude their use in portable systems. In addition, higher-frequency optical scanning could enable new applications that are not practical with conventional scanning mirrors, such as raster-scanning projection video displays [1], and would significantly improve the performance of scanning mirrors in existing applications, such as laser printers. MFMS optical scanners promise to enable these new applications, and dramatically reduce the cost of optical systems.

However, previously demonstrated MEMS mirrors have not been able to simultaneously meet the requirements of high scan speed and high resolution. A plethora of micromirror designs have been presented, but none has been able to satisfy the potential of MEMS: a high-speed, high-performance scanning mirror. For example, surface-micromachined scanning mirrors actuated with electrostatic combdrives have been shown to operate at high scan speeds (up to $21 \mathrm{kHz}$ ), but static and dynamic mirror deformation limits the resolution to less than $20 \%$ of the diffraction-limited resolution. Magnetically actuated mirrors have been demonstrated with high speed and large amplitude [2], but have not demonstrated high resolution, and often require off-chip actuation.

In order to take advantage of the unique capabilities of micromachining technology, we have fabricated a new type of micromirror capable of high-speed, high-resolution scanning with low power consumption.

\section{THEORY OF OPERATION}

\section{STEC Micromirror}

Figure 1 shows a schematic of the Staggered Torsional Electrostatic Combdrive (STEC) micromirror, which is made from two layers of single-crystal silicon separated by a $1.7 \mu \mathrm{m}$-thick silicon dioxide layer. The mirror, torsion hinge, and moving comb teeth are in the top silicon layer, and the fixed comb teeth are in the bottom silicon layer. Applying voltage between the top and the bottom layers attracts the moving comb teeth to the fixed comb teeth, thus exerting torque on the mirror and causing it to tilt. The torsion hinges, which are anchored to the bottom silicon layer, provide restoring torque. Similar torsional actuators have been

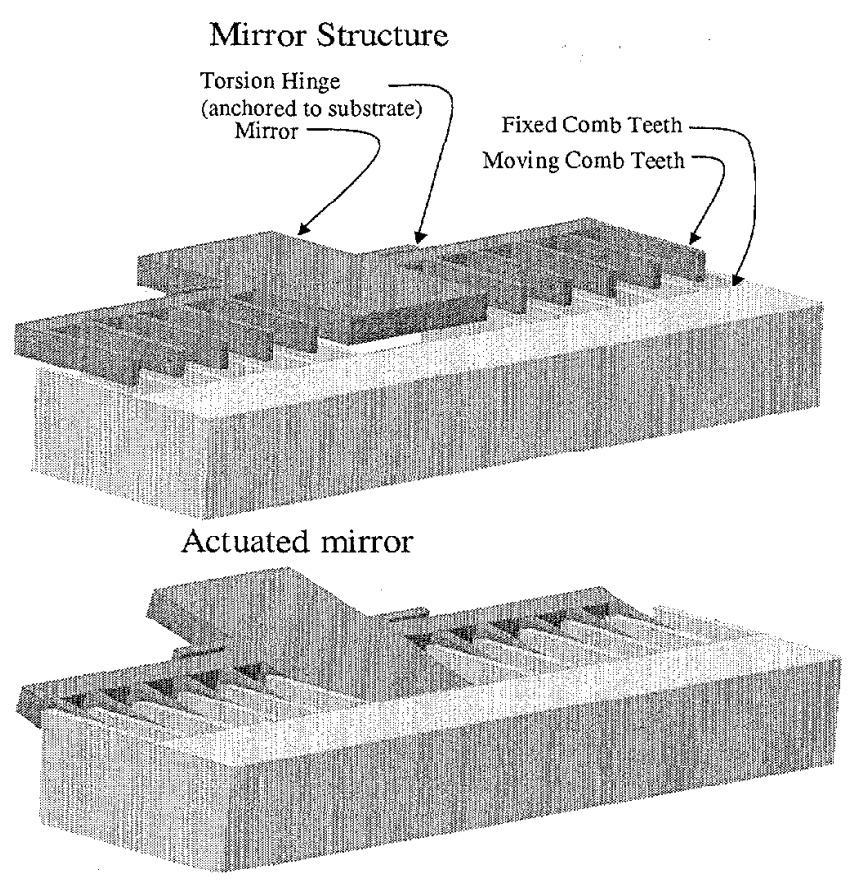

Figure 1. Schematic of a STEC micromirror. Applying a voltage between the top and the bottom layers generates a torque on the mirror, thereby causing it to tilt.

described earlier [3][4]. SEMs of the completed STEC micromirror are shown in Figure 2.

This torsional electrostatic combdrive offers several advantages over other electrostatic-actuator designs. First, the actuator applies torque to the mirror directly - there are no hinges to couple linear motion of an actuator into torsional mirror motion. This greatly simplifies the design of the structure, and makes postfabrication assembly steps unnecessary.

Second, the actuator starts in an unbalanced state and is capable of static mirror positioning as well as resonant scanning. Previously demonstrated balanced torsional electrostatic actuators have been very promising for resonant operation [5], but are not capable of static mirror positioning.

Third, the torsional combdrive offers an advantage over gapclosing actuators because the energy density in the combdrive is higher than that in a gap-closing actuator, thereby allowing larger scan angles at high resonant frequencies.

\section{Optical Resolution}

The optical resolution - defined as the ratio of the opticalbeam divergence and the mirror scan angle - is an essential performance metric for a scanning mirror. For a perfectly flat mirror under uniform illumination, the farfield intensity distribution is an Airy pattern, which has a full-width-half-max half-angle beam divergence $\alpha$ (the resolution criteria used for video displays [6]) given by

$$
\alpha=\frac{1.03 \lambda}{D}
$$




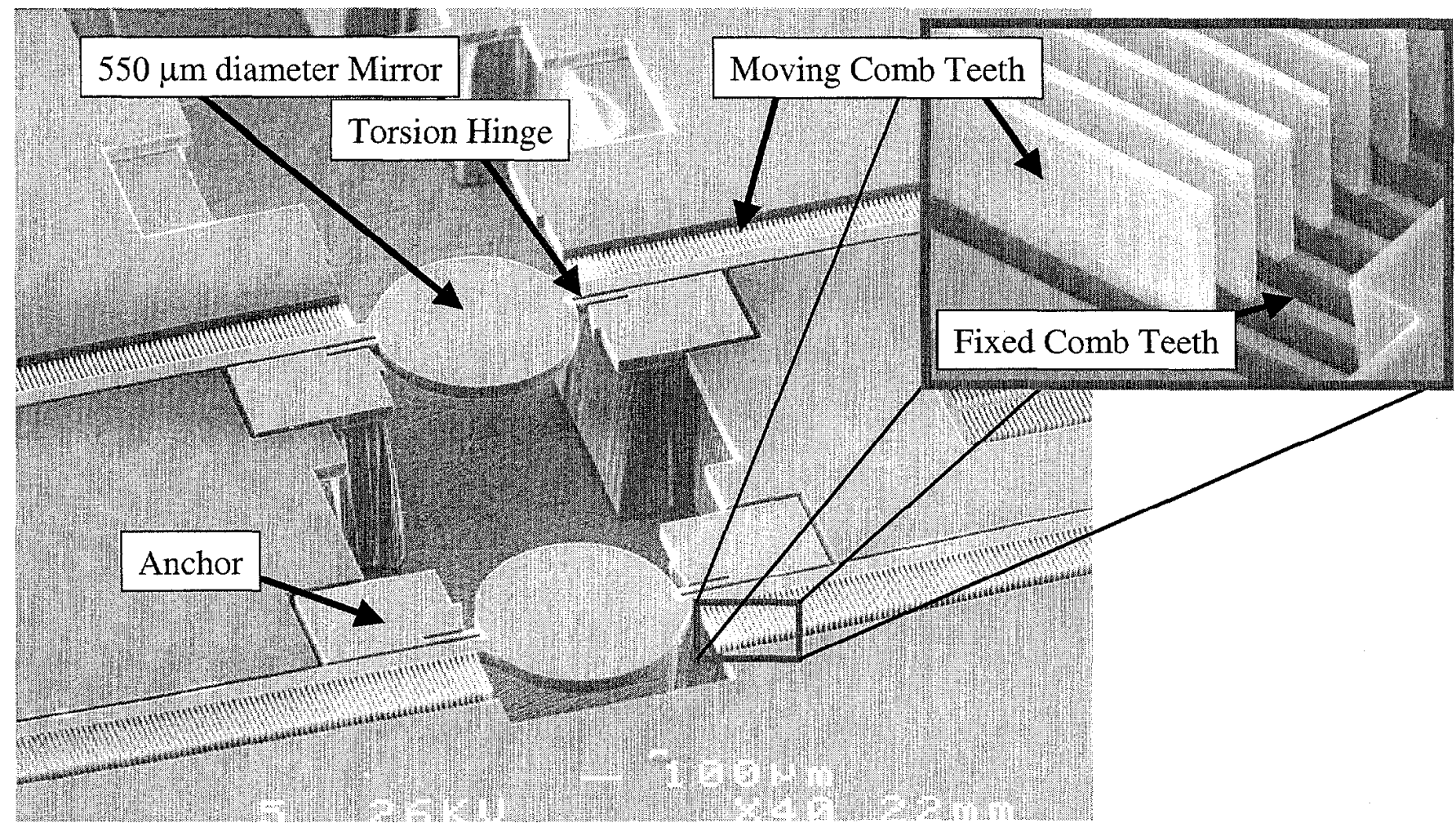

Figure 2. SFM of two STEC micromirrors. The SEM chuck is visible through the wafer below the mirror. The inset SEM in the upper right shows a close-up view of the fixed and moving combteeth.

where $\lambda$ is the wavelength of the incident light, and $D$ is the mirror diameter. The resulting optical resolution $N$ is

$$
N=\frac{4 \theta}{\alpha}=\frac{4 \theta D}{1.03 \lambda}
$$

where $\theta$ is the mechanical half-angle mirror scan (the total optical scan is $4 \theta$ ).

Dynamic mirror deformation can also contribute to beam divergence, thereby decreasing the optical resolution. For a mirror where the torsion hinge is the dominant compliance, the nonplanar surface deformation $\delta$ of a rectangular scanning mirror of halflength $L$ with angular acceleration $(2 \pi f)^{2} \theta$ (where $f$ is the scan frequency) is [7]

$$
\delta=0.183 \frac{\rho\left(1-v^{2}\right)(2 \pi f)^{2} \theta}{E t^{2}} L^{5}
$$

where $\rho$ is the material density, $v$ is Poisson's ratio, $E$ is Young's modulus, and $t$ is the mirror thickness.

The Rayleigh limit, the maximum amount of surface deformation tolerable without significant degradation in image quality, allows a peak-to-valley surface deformation of $\lambda / 4$ [8].

For a $550 \mu \mathrm{m}$-long ( $275 \mu \mathrm{m}$-half-length) rectangular single-crystalsilicon mirror of thickness $50 \mu \mathrm{m}$, half-angle mechanical scan $6.25^{\circ}$, and resonant frequency $34 \mathrm{kHz}$, the calculated dynamic deformation is $8 \mathrm{~nm}$ - much lower than the Rayleigh limit for 655 $\mathrm{nm}$ light $(164 \mathrm{~nm})$. For comparison, a $550 \mu \mathrm{m}$-long surfacemicromachined mirror of thickness $1.5 \mu \mathrm{m}$ maintains the surface flatness within the Rayleigh limit only up to a frequency of 4.6 $\mathrm{kHz}$.

\section{FABRICATION}

The STEC micromirrors are fabricated using deep reactiveion etching and a bond-and-etchback process with a buried pattern. The process flow is shown in Figure 3.

First, a silicon wafer is oxidized in steam at $1000^{\circ} \mathrm{C}$ to grow $0.2 \mu \mathrm{m}$ of thermal oxide. This wafer is patterned with the BURIED pattern, and $100 \mu \mathrm{m}$-deep trenches are etched into the wafer using an STS deep reactive-ion etcher to form the fixed combteeth. This wafer, along with another wafer having $1.5 \mu \mathrm{m}$ of thermal oxide, is cleaned and the two wafers are bonded together with the fixed combteeth at the wafer interface. The bonded wafer pair is then annealed at $1100^{\circ} \mathrm{C}$ for one hour to increase the bond strength [9].

Next, the bonded wafer is ground and polished to leave a 50 $\mu \mathrm{m}$-thick layer of silicon above the oxide interface. The resulting silicon-on-insulator (SOI) wafer is oxidized at $1100^{\circ} \mathrm{C}$ in a steam ambient to form a $1.1 \mu \mathrm{m}$-thick oxide layer on the top and bottom. Alignment between the subsequent patterns and the buried combtecth is achicved by etching a window in the top-laycr silicon above the two dice used to align the mask to the wafer. These two dice are sacrificed to achieve the necessary alignment precision between the moving combteeth (on the top layer) and the fixed combteeth (on the bottom layer).

The FRONTSIDE pattern, which defines the moving comb teeth, the mirror, and the torsion hinges, is then patterned and etched into the top oxide layer (the pattern is etched into the silicon in a later step). The alignment of this step is critical because misalignment between the moving combteeth and the fixed combteeth can lead to instability in the torsional combdrive. We have typically been able to achieve better than $0.2 \mu \mathrm{m}$ alignment between the BURIED pattern and the FRONTSIDE 
Etch wafer1

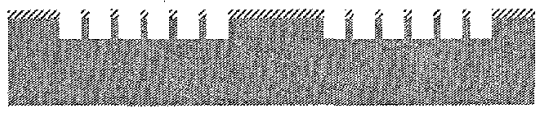

\section{Bond wafer1 and wafer2}

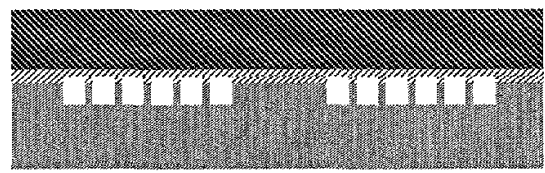

\section{Grind and \\ polish wafer2}

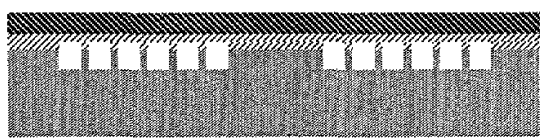

\section{Backside etch wafer1}

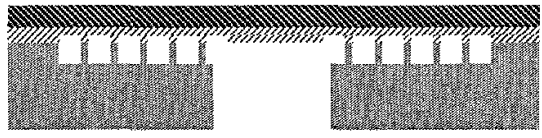

\section{Pattern and etch wafer 1}

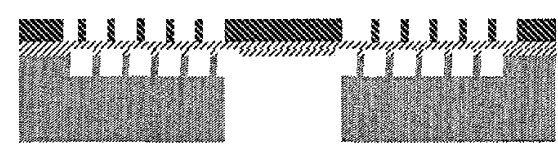

Release

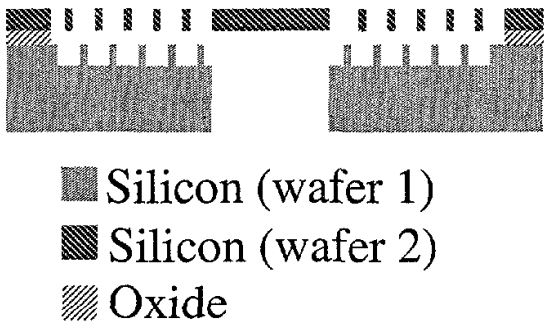

Figure 3. STEC micromirror fabrication process flow.

pattern using a GCA i-line wafer stepper, and this alignment error has not limited the STEC micromirror performance.

Next, the oxide and silicon on the backside of the bottom wafer are patterned with the HOLE layer, and the entire bottom wafer is etched to open an optical path underneath the micromirror. The silicon on the top layer is then etched using the previously patterned top oxide layer as an etch mask. The structure is released with a timed HF etch to remove the sacrificial oxide film below the combteeth and mirror. Finally, a $100 \mathrm{~nm}$ thick aluminum film is evaporated onto the bottom of the mirror to increase the reflectivity for visible light to approximately $92 \%$ [10].

\section{RESULTS}

The STEC mirror excels in all of the critical performance criteria: cost, resolution, scan speed, scan repeatability, size, power consumption, and reliability. This section discusses measurements of four of these performance criteria for one STEC mirror design.

\section{Resolution}

The surface deformation of the micromirror was characterized using the stroboscopic interferometer reported in [11]. Figure 4 shows the dynamic deformation of the micromirror at the end of the scan where the dynamic deformation reaches its maximum. The total deformation is less than $30 \mathrm{~nm}$, considerably below the Rayleigh limit, and does not significantly reduce the optical resolution.

Figure 5 shows a photograph of the scan of a STEC mirror with a $655 \mathrm{~nm}$ laser pulsed synchronously with the mirror scan to generate 43 spots. The mirror scan is sinusoidal, so the timing of the laser pulses was adjusted across the scan to produce equally spaced spots. Figure 5 also shows a close-up image of three individual spots captured with a CCD camera. By fitting a Gaussian curve to each of these three spots, we were able to determine the full-width-half-max beam width and the distance between the spots. The spot size and separation at eight different regions across the scan give the measured total optical resolution of 350 pixels. The resolution of this $550 \mu \mathrm{m}$-diameter mirror with $24.9^{\circ}$ optical scan and $655 \mathrm{~nm}$ laser light is near the diffractionlimited resolution of 355 pixels from Eq. [2].

\section{Scan Speed}

STEC micromirrors have been demonstrated with diameters of $550 \mu \mathrm{m}$ and resonant frequencies up to $42 \mathrm{kHz}$ - almost an order of magnitude faster than commercially available optical scanners. Larger STEC mirrors have also been fabricated (up to 2 $\mathrm{mm}$ ) with lower resonant frequencies. Figure 6 shows the frequency response of a $550 \mu \mathrm{m}$-diameter micromirror.

The main limitation of macro-scale scanners comes from the dynamic deformation described by Eq.[3] - the dynarnic deformation scales as the fifth power of the mirror length, so large mirrors scanning at high speeds will have considerable dynamic deformation. For example, a $10 \mathrm{~mm}$-diameter, $1 \mathrm{~mm}$-thick mirror with a mechanical scan of $\pm 6.25^{\circ}$ maintains less than $164 \mathrm{~nm}$ dynamic deformation (the Rayleigh limit for $655 \mathrm{~nm}$ light) up to a frequency of only $2.2 \mathrm{kHz}$. Large-scale mirrors cannot achieve the speeds demonstrated with the STEC micromirrors withoul severe dynamic deformation or very thick mirrors.

\section{Power Consumption}

High-speed scanners require more torque than low-speed scanners to reach the same scan angle. In order to generate the torque necessary for large angle, high-frequency operation of the STEC micromirror, we use fairly high voltages. The $550 \mu \mathrm{m}-$ diameter mirror with resonant frequency $34 \mathrm{kHz}$ requires a 171 Vrms input sine wave for a total optical scan of $24.9^{\circ}$. To simplify mirror testing and operation, we use a small $\left(1 \mathrm{~cm}^{3}\right)$ 25:1 transformer, allowing us to use a conventional $0-10 \mathrm{~V}$ function generator to drive the scanning mirrors with a sinusoidal waveform of amplitude up to $250 \mathrm{~V}$. The use of the transformer also provides cfficient power conversion, so the power consumption of the entire system can be much lower than systems requiring highvoltage power supplies and opamps.

This power consumption is the sum of the power dissipation in the drive electronics and the power dissipated by air and material damping. The power consumption due to damping is

$$
P=\frac{1}{2} b \theta^{2} \omega^{2}=\frac{1}{2} \frac{k}{Q} \theta^{2} \omega
$$

where $k$ is the torsional spring stiffness, $b$ is the torque damping factor, $\theta$ is the mechanical scan half angle (the total optical scan is $\left.\pm 2 \theta_{0}\right), \omega$ is the resonant frequency, and $Q$ is the resonant quality factor. For the $34 \mathrm{kHz} 550 \mu \mathrm{m}$-diameter mirror scanning $25^{\circ}$ optical $\left( \pm 6.25^{\circ}\right.$ mechanical $)$, the calculated stiffness $k=3.93 \times 10^{-5} \mathrm{Nm} / \mathrm{radian}$, the measured resonant quality factor $\mathrm{Q}=273$, so the power consumption due to damping from Eq. [4] is $0.18 \mathrm{~mW}$. Vacuum packaging can be used to reduce the viscous damping, and thereby decrease the power consumption.

The measured power consumption is $6.8 \mathrm{~mW}$, indicating that the majority of the power consumption is in charging and discharging the parasitic capacitance and losses in the transformer power conversion. 

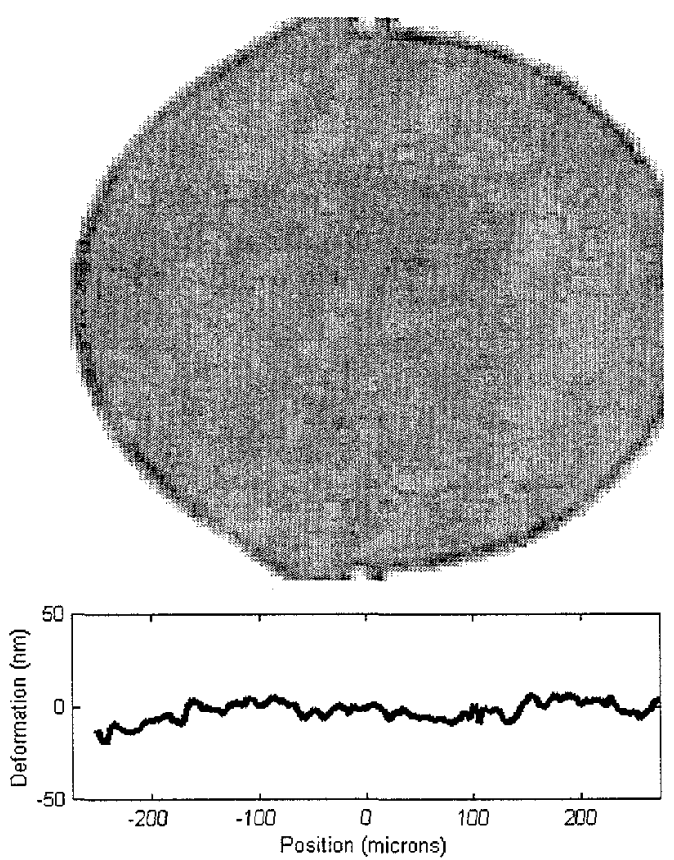

Figure 4. Static and dynamic deformation of the $550 \mu \mathrm{m}$ diameter mirror is less than $30 \mathrm{~nm}$. The graph below the grayscale surface height map shows the measured mirror deformation along the center of the mirror perpendicular to the torsion hinges.

\section{Reliability}

The STEC micromirror is expected to be extremely reliable due to its simple structure. It is predicted that the failure point for the structure will be the torsion hinges (at the point of highest strain). The maximum strain in a $50 \mu \mathrm{m}$-thick, $15 \mu \mathrm{m}$-wide, 150 $\mu \mathrm{m}$-long hinge (the hinge used for the $550 \mu \mathrm{m}$-diameter mirror wilh resonanl frequency of $34 \mathrm{kHz}$ ) with a total scan of $\pm 6.25^{\circ}$ is approximately $1.8 \%$. Mirrors have been operated at this level for over 200 million cycles without any noticeable degradation in performance, but this strain may be too high for acceptable long. term reliability. Future designs will use wider, longer hinges to reduce the maximum strain while retaining the same stiffness.

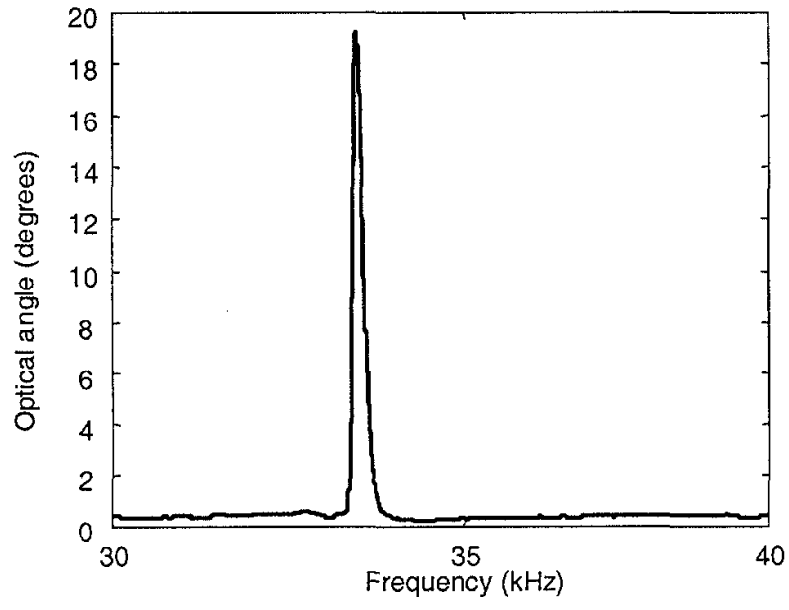

Figure 6: Frequency response of STEC micromirror.

\section{CONCLUSIONS}

The potential of micromachined mirrors over conventional scanning mirrors - high scan speed, small size, and low cost with diffraction-limited optical performance - has been demonstrated

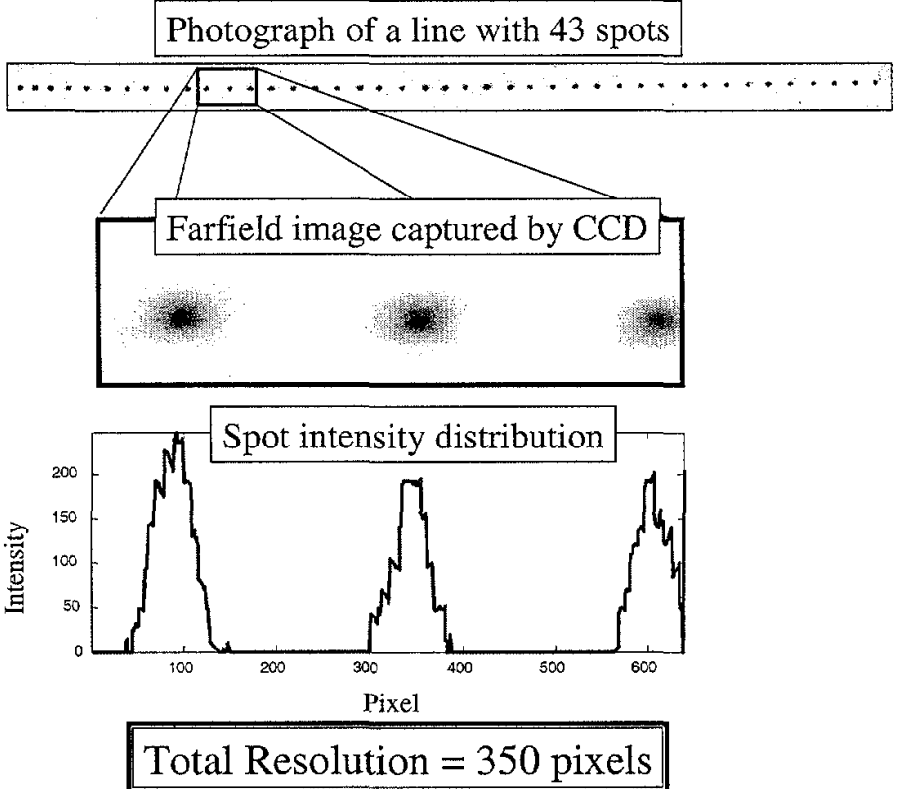

Figure 5. Optical scan of STEC micromirror operating at $34 \mathrm{KHz}$, with close-up image of spots and intensity distribution plot. The images recorded on the photograph and the $C C D$, which were red-onblack, were converted to black-on-white for this figure.

with the Staggered Torsional Electrostatic Combdrive (STEC) micromirror. The scan speed of the STEC micromitror is difficult to achieve with large-scale optical scanners, and exceeds the performance of previously demonstrated micromachined scanning mirrors.

\section{REFERENCES}

1. R.A. Conant, P.M. Hagelin, U. Krishnamoorthy, O. Solgaard, K.Y. Lau, R.S. Muller, "A Full-Motion Video Display Using Micromachined Scanning Micromirrors," proc. Transducers '99, Sendai, Japan (1999), pp 376-379.

2. A. Garnier, T. Bourouina, H. Fujita, T. Hiramoto, E. Orsier, J-C. Peuzin, "Contactless actuationof bending and torsional vibrations for $2 \mathrm{~d}$-opticalscanner application," proc. Transducers '99, Sendai, Japan (1999), pp. 1876-1877.

3. Z.J. Yao, N.C. MacDonald, N.C. "Single crystal silicon supported thin film micromirrors for optical applications," Optical Engineering, 36 (no.5), pp. 1408-13 (1997).

4. J.-L.A Yeh, J. Hongrui; N.C. Tien, "Integrated polysilicon and DRIE bulk silicon micromachining for an electrostatic torsional actuator.", JMEMS, 8 (no.4), pp. 456-65 (1999)

5. H. Schenk, P. Durr, D. Kunze, H. Lakner, H. Kuck, "An electrostatically excited 2D-micro-scanning-mirror with an in-plane configuration of the driving electrodes," proc. MEMS 2000, Miyazaki, Japan (2000), pp. 473478.

6. J. Hagerman, "Optimum spot size for raster-scanned monochrome CRT displays", Journal of the SID, 1 (no.3), pp. 367-369 (1993)

7. P.J. Brosen, "Dynamic Mirror Distortions in Optical Scanning," Applied Optics Vol. 11, No. 12, pp. 2987-2989, 1972.

8. D.J. Jacobs, Fundamentals of Optical Engineering, 1st ed. New York: McGraw-Hill, 1943.

9. W.P. Maszara, G. Goetz, A. Caviglia, and J.B. McKitterick,

"Bonding of silicon wafers for silicon-on-insulator," Journal of Applied Physics, 64(10), pp. 4943-4950 (15 November 1988).

10. G.R. Elion, H.A. Elion, Electro-Optics Handbook, New York: Marcel Dekker, Inc., 1979.

11. M. Hart, R.A. Conant, K.Y. Lau, R.S. Muller, "Time-resolved measurement of optical MEMS, using strohoscopic interferometry, " proc. Transducers '99, Sendai, Japan (June 1999), pp. 470-473. 